\title{
Rehabilitation of an atrophic jaw with three short implants and a customized
}

\section{milled bar overdenture}

António Sérgio Silva ${ }^{1}$, Juliana de Sá ${ }^{2}$, Carlos Manuel Aroso ${ }^{1}$, Eduardo Santos ${ }^{3}$, Luís Neves², and José Manuel Mendes ${ }^{1}$

1. Department of Dental Sciences, CESPU, Institute of Research and Advanced Training in Health Sciences and Technologies, Portugal

2. Department of Oral Rehabilitation, Instituto Universitário de Ciências da Saúde, Portugal

3. Department of Fixed Dental Prosthesis, Dentalmaia Laboratory, Portugal

\section{CASE STUDY}

Please cite this paper as: Silva AS, Sá J, Aroso C, Santos E, Neves L, Mendes JM. Rehabilitation of an atrophic jaw with three short implants and a customized milled bar overdenture. AMJ 2018;11(12):565-572.

https://doi.org/10.21767/AMJ.2018.3537

\section{Corresponding Author:}

António Sérgio Silva

Department of Dental Sciences, CESPU,

Institute of Research and Advanced Training in Health

Sciences and Technologies, Portugal

Email: asergio.silva@iucs.cespu.com

\section{ABSTRACT}

Edentulism is frequently observed in older individuals, and edentulous patients with conventional prostheses have reported lack of retention, support, stability, and chewing and phonation failures. Overdentures are a good option for rehabilitation of edentulous patients. Two or more implants supporting a milled bar with attachments may be used for rehabilitation of these patients, since this arrangement allows very limited rotation and vertical movement, thereby increasing patient satisfaction. This report describes a clinical case involving oral rehabilitation of an atrophic jaw with three short implants and a customized milled bar overdenture.

\section{Key Words}

Oral rehabilitation, short implants, overdentures, milled bar, attachments, atrophic jaws

\section{Implications for Practice:}

\section{What is known about this subject?}

Implant-supported rehabilitation of atrophic mandibles with severe bone resorption with both surgical and prosthetic procedures is challenging due to the high risk of mandible fracture.

\section{What new information is offered in this case study?}

After short-implant placement, production of a customized milled bar adapted to the patient's degree of mandibular resorption can more successfully cancel the disinsertion force of the prosthesis.

3. What are the implications for research, policy, or practice?

Rehabilitation of an atrophic jaw with short implants and a combination of different attachment systems can greatly increase the overdenture retention capacity.

\section{Background}

Edentulism is an oral health problem that is frequently encountered in older individuals and is known to impair masticatory function. ${ }^{1}$ Dental implants have revolutionized the treatment of edentulous patients, with conventional dental prostheses being an alternative in many rehabilitation treatments. ${ }^{2}$ Overdentures supported by two implants are recognized as the primary treatment option for edentulous patients. ${ }^{3,4}$ However, overdentures are subject to complex three-dimensional movements as a result of the masticatory load. ${ }^{5-9}$

A large number of retainer systems to be used between the prosthesis and the implant are currently available in the market, which can make the choice of a connection system complex for the dentist. At present, these choices and the resultant designations are fundamentally based on opinions and clinical experience rather than actual tests and scientific 
results. $^{6}$

An attachment is a mechanical device that allows retention, fixation, and stabilization of a dental prosthesis. ${ }^{7}$ Attachments can be divided according to their configuration (bar or individual) or their capacity of movement (resilient or rigid). ${ }^{8}$ Implant-supported overdenture attachments are expected to show sufficient retention to improve the stability of the prosthesis, while also allowing easy insertion and disinsertion of the prosthesis for the patient. ${ }^{6-11}$

The use of short implants may be indicated in cases without optimal bone availability. Short implants may also be recommended in several situations to avoid costly and timeconsuming local bone augmentation procedures. Notably, fatigue fracture is a type of late failure that may appear after long periods of use. Therefore, the manufacturer's guidelines for the use of short implants should be taken into account. $^{12}$

\section{Case details}

A 65-year-old Caucasian female patient visited our dental practice with complaints regarding her lower total acrylic prosthesis. The patient had received a lower total acrylic prosthesis in 2006 and had persistent complaints related to the mobility of the prosthesis (constant but inefficient use of adhesive), which caused intraoral ulcers and constant painful areas in the lips and difficulties in chewing and phonation that had aggravated over the last two years. To minimize the pain caused by the prosthesis, the patient had developed involuntary and unreflective movements of the tongue, mucous membranes, and lips over the years. Therefore, she sought treatment to improve the efficiency of retention and mastication of her total inferior prosthesis.

The patient's general health status included elevated cholesterol levels and hypertension, for which she was receiving medication. She underwent orthopantomography, computed tomography, intra- and extra-oral photography, and clinical examination. The intraoral examination showed a completely edentulous mandible with a smooth, highly reabsorbed crest. She did not report any complaint regarding maxillary partial denture retention. However, the examinations showed severe diminution of the vertical dimension with little labial support and ulcers in the vestibule zone.

The treatment started with a wear of the prosthesis to relieve the ulcerated areas, avoidance of prosthesis use for as long as possible, and application of Elugel ${ }^{\circledR} 3$ to 4 times a day and mouthwashes with chlorhexidine 0.12 per cent 2 times per day. An X-ray examination showed dense compact bone in the anterior mandibular region. The CT scan revealed a distance of approximately $30 \mathrm{~mm}$ between the mental foramens with $10 \mathrm{~mm}$ of bone height and visible signs of sharp vertical resorption (Figure 1). Implant placement planning was performed using Blue Sky Pan ${ }^{\circledR}$ software. The patient was in good health and her blood reports were checked.

Under antibiotic prophylaxis and a standard aseptic protocol, nerve block and infiltration anaesthesia were administered. A full-thickness crestal incision bisecting the keratinized gingiva from the first premolar to first premolar was made, and a mucoperiosteal flap was created. Three short implants (Megagen Anyridge ${ }^{\circledR}, 4.0 \times 8.5 \mathrm{~mm}$, Megagen Daegu, Korea) were then distributed across the space between the mental foramens according to the protocol described by the manufacturer. Cover screws and silk sutures were applied to the surgical area at the end. The patient's existing prosthesis was rebased with a tissue conditioner and replaced in the mouth (Figure 2).

Three months later, a clinical and radiographic evaluation of the implants was performed. The implants had perfectly osseointegrated with good stability without any inflammatory signs, and the patient showed a healthy and keratinized gingiva. Radiographic assessments showed no signs of bone loss or the existence of any perimplant inflammatory process. The implants were therefore ready to undergo the prosthetic phase (Figure 3 ).

In the second phase, we exposed and removed the cover screws and replaced them with healing caps over a twoweek period. At the end of this period, impressions of the three implants were obtained using impression copings for a closed tray (Figure 4).

The prosthetic phase began with an impression directly to the implants, using a standard tray, three closed tray impression copings, and a mixture of silicone putty and light (Figure 5). The laboratory was required to send a screw-in occlusion wax to the implant to determine the intermaxillary relationship of the patient and to resize the patient's vertical dimension, which was greatly diminished (Figure 6).

In order to determine the intermaxillary relationship, we used the Willis metric method, which uses a ruler with an adjustable cursor, designed by the author and called the Willis compass, to record the distance from the outer corner of the eye to the labial commissure and decreases, 
arbitrarily, about 3 to $4 \mathrm{~mm}$ equivalent to the free functional space to establish the height at which rehabilitation will be performed. A plastic fork, OneBite ${ }^{\circledR}$ System (Precision Dental Products, London, England), was also used for bite registration and registration of the patient's midline and horizontal lines. These records were sent to the laboratory and transferred to a semi-adjustable articulator (Figure 7).

One week later, a teeth test was performed to confirm that the occlusion, colour, phonation, and vertical dimension were in harmony with the patient's facial and dental parameters. After obtaining approval in this important step and analysing the available space underlying the future prosthesis, the projection and fabrication of the individualized milled bar began (Figure 8).

In this process, a chrome-cobalt bar was prepared with height and thickness of $10 \mathrm{~mm}$ and $5 \mathrm{~mm}$, respectively, and two Equator ${ }^{\circledR}$ OT attachments (Rhein 83, Bologne, Italy) on the bar and two vertical Ceka ${ }^{\circledR}$ Preci-line attachments (Alphadent NV, Waregen, Belgium) at the rear ends of the bar were added so as to cancel the horizontal forces and the vertical forces of disinsertion caused by chewing. After waxing (Yeti dental, Engen, Germany) and casting of the bar (Gialloy PA, SRL Dental GmbH, Ludwigshafen, Germany), an adaptation test was performed in the mouth with radiographic control of the passive settlement (Figure 9).

Finally, when the setting of the bar was adequate, molten reinforcement of the future prosthesis was performed to minimize the risk of fracture. This process consisted of waxing and casting to obtain a chromium-cobalt structure (Gialloy PA, SRL Dental GmbH, Ludwigshafen, Germany) underlying the prothesis and acrylics (Figure 10).

Clinically, the process ended with the placement of the bar and the tightening of the implant screws at $35 \mathrm{Nm}$, placement and adaptation of the overdenture, verification of the occlusion, verification of the retention of the prosthesis in the mouth, relief of pressure zones, and maintenance and hygiene advice for the entire structure (Figure 11).

\section{Discussion}

Rehabilitation of an atrophic jaw is one of the most difficult procedures in dental medicine. The low bite makes it difficult to retain conventional prostheses and also precludes the use of osseointegrated implants. Several techniques for osseointegration in these bone defects have been described in the literature. Most of these techniques seek to reconstruct the reabsorbed alveolar process.
Osteogenic distraction with distractors, especially in these cases, yields an average gain of $9 \mathrm{~mm}$. Another technique described is that of tissue expansion. However, all of these reconstructive techniques involve high morbidity and long treatment durations. ${ }^{13}$

The term "short implant" is subjective with some lack of consensus in the literature regarding its definition. However, since the minimum length for proven predictable success has always been $10 \mathrm{~mm}$, implants of this length are normally considered standard length and, therefore, any implant below $10 \mathrm{~mm}$ is referred to as a short implant. ${ }^{14-16}$ Currently, short implants present results similar to those obtained with larger implants. These success rates are mainly due to improvements in surfaces that increase bone/implant contact. ${ }^{17-20}$

In addition to the acceptable success rate described in the literature, these implants also cause lower morbidity and require shorter treatment durations in relation to reconstructive techniques, which is relevant since the majority of the patients presenting with this condition have advanced age and systemic health problems. ${ }^{17-20}$

Schwindling et al. proposed a study whose main objective was to evaluate the survival rate of short implants as well as to evaluate the complications and need for maintenance of overdentures placed on them. ${ }^{21}$ In that study, 99 short implants were placed in 25 patients with an average age of 72 years. Eight of these implants were considered lost in the osseointegration phase, and two were fractured at the time of the surgical procedure. Thus, in the first seven years, a survival rate of 92 per cent was determined with a clear advantage, in terms of success, for the complete dentures (94.9 per cent) over the partial dentures (81 per cent). ${ }^{21}$ Therefore, the authors concluded that the survival rate of the short implants was good and very close to the values found in current literature for longer implants. ${ }^{21}$

Even in assessments of the level of stress applied on short implants, the current literature reports that in most cases, the values are very similar to those found in the longer implants. ${ }^{21}$ Misch CE., in a literature review of short dental implants, found that any increase in implant length, such as the use of $10 \mathrm{~mm}$ implants instead of $7 \mathrm{~mm}$ implants, may not provide any significant improvement in anchorage. ${ }^{22}$ In fact, the diameter of the implants plays a more relevant role in the distribution of occlusal forces than its length. ${ }^{22-24}$ In analyses of the stress levels on short implants, the literature shows that when placed in a bone of adequate density, the greatest magnitude of stress is concentrated in the first 
cervical $5 \mathrm{~mm}$ of the bone-implant interface, similar to the values noted for implants of greater length. ${ }^{22-24}$

Peixoto et al. studied the use of short vertical implants or with several inclinations to evaluate the distribution of perimplant stress. For this purpose, the authors created six similar models of an atrophic mandible with $8 \mathrm{~mm}$ in height. To evaluate the influence of the length factor, the authors used two types of implants with distinct heights: short implants measuring $7 \mathrm{~mm}$ and conventional implants measuring $9 \mathrm{~mm}$. To evaluate the influence of the angulation factor, the authors used three different configurations: the first used four implants inclined at a 45-degree angle, the second used two inclined and two vertical implants, and the third used four vertical implants. ${ }^{25}$ The authors concluded that the rehabilitation of atrophic jaws with four short implants placed at a 45-degree angle generated less stress than longer implants with transcortical involvement and implants placed in a vertical position. ${ }^{25}$

Pimentel et al. conducted a study with the main objective of comparing the level of stress in atrophic jaws when rehabilitated with implants of conventional length and diameter or with short implants. For this purpose, they designed several configurations with two diameters (4 and $5 \mathrm{~mm}$ ) and three different lengths $(5,7$ and $9 \mathrm{~mm})$. As a result, the level of stress in narrow implants increased significantly as the implant length decreased. The same authors verified that when the diameter increased, this difference no longer existed between short implants and longer implants. Thus, they concluded that under certain conditions, both short and longer implants may present similar biomechanical behaviour. ${ }^{26}$

By reviewing the current literature, we can affirm that short implants present a viable alternative to the rehabilitation of atrophic jaws, often allowing clinicians to avoid complex surgical procedures and the consequent prolonged periods of healing, painful postoperative recovery, and increased morbidity. ${ }^{20-27}$

Improvement in the surface treatment of implants has been found to be essential for increasing the confidence in the application of such short implants without using advanced surgical techniques such as mandibular nerve transposition or paranasal sinus elevation and grafting. ${ }^{20-27}$ This simplification of the processes also benefits the patient in economic terms, as they will have to spend much fewer resources on a solution that allows them to recover their masticatory capacity, phonation, and facial aesthetics. ${ }^{20-27}$ The current literature includes studies in which overdentures made over short implants, as in this case, showed high success rates similar to those obtained with conventional-sized implants, while also showing a high degree of patient satisfaction in terms of retention and reliability. $^{20-27}$

\section{Conclusion}

Rehabilitation of an atrophic jaw with three short implants and a customized milled bar overdenture, as described in this article, allows masticatory functionality and comfort to be restored in such cases. The major practical contribution of the present clinical case was that it demonstrated a good prosthetic solution in more adverse situations. In future investigations, it will be important to perform a long-term follow-up to an atrophied edentulous mandible with short implant-supported overdenture, to verify its reliability over the years.

\section{References}

1. Al Nawas B, Brägger $U$, Meijer HJA, et al. A double blind randomized controlled trial (RCT) of titanium 13Zirconium versus titanium grade IV small diameter bone level implants in edentulous mandibles- Results from a 1 year observation period. Clin Implant Dent Relat Res. 2012;14(6):896-904.

2. Carlsson GE. Early in contrast to recent methods to evaluate masticatory function in implant patients. J Prosthodont Res. 2012;56(1):3-10.

3. Silva AS, Aroso C, Ustrell $R$, et al. The influence of saliva $\mathrm{pH}$ value on the retention and durability of bar-clip attachments. J Adv Prosthodont. 2015;7(1):32-8.

4. Aroso C, Silva AS, Ustrell R, et al. Effect of abutment angulation in the retention and durability of three overdenture attachment systems: An in vitro study. J Adv Prosthodont. 2016;8:21-9.

5. Kobayashi M, Srinivasan M, Ammann P, et al. Effects of in vitro cyclic dislodging on retentive force and removal torque of three overdenture attachment systems. Clin Oral Implants Res. 2014;25(4):426-34.

6. Jabbour Z, Fromentin O, Lassauzay C, et al. Effect of implant angulation on attachment retention in mandibular two-implant overdentures: A clinical study. Clin Implant Dent Relat Res. 2014;16(4):565-71.

7. Sá J, Silva AS, Aroso C, et al. Degree of patient satisfaction with overdentures. Int J Sci Res (Ahmedabad). 2017;6(7):518-522.

8. Abi Nader S, de Souza R, Fortin D, et al. Effect of simulated masticatory loading on the retention of stud attachments for implant overdentures. J Oral Rehabil. 2011;38(3):157-64.

9. Trakas T, Michalakis K, Kang K, et al. Attachment systems 
for implant retained overdentures: a literature review. Implant Dent. 2006;15(1):24.

10. Fromentin O, Lassauzay C, Nader SA, et al. Wear of matrix overdenture attachments after one to eight years of clinical use. J Prosthet Dent. 2012;107(3):191-8.

11. Kim MS, Yoon MJ, Huh JB, et al. Implant overdenture using a locator bar system by drill and tapping technique in a mandible edentulous patient: a case report. J Adv Prosthodont. 2012;4(2):116-20.

12. Zinsli B, Sagesser T, Mericske E, et al. Clinical Evaluation of Small-Diameter ITI Implants: A Prospective Study. Int J Oral Maxillofac Implants. 2004;19:92-99.

13. Raghoebar GM, Heydenrijk K, Vissink A. Vertical distraction of the several resorbed mandibles. The Groningen distraction device. Int J Oral Maxillofac Surg. 2000;29:416-420.

14. das Neves FD, Fones D, Bernardes SR, et al. Short implants: an analysis of longitudinal studies. Int J Oral Maxillofac Implants. 2006;21:86-93.

15. Gentile MA, Chuang SK, Dodson TB. Survival estimates and risk factors for failure with $6 \times 5.7-\mathrm{mm}$ implants. Int J Oral Maxillofac Implants. 2005;20:930-7.

16. Morand M, Irinakis T. The challenge of implant therapy in the posterior max- illa: providing a rationale for the use of short implants. J Oral Implantol. 2007;33:257-66.

17. Anitua E, Orive G, Aguirre JJ, et al. Five-year evaluation of short dental implants placed in posterior areas: A retrospective study. J Periodontol. 2008;79:42-8.

18. Grant BTN, Pancko FX, Kraut RA. Outcomes of placing short dental implants in the posterior mandible: $A$ retrospective study of 124 cases. J Oral Maxillofac Surg. 2009;67:713-7.

19. Rossi F, Ricci E, Marchetti C, et al. Early loading of single crowns supported by $6-\mathrm{mm}$-long implants with a moderately rough surface: a prospective 2-year followup cohort study. Clin. Oral Impl Res. 2010;21:937-43.

20. Tawil G, Younan R. Clinical evaluation of short, machined-surface implants followed for 12 to 92 months. Int J Oral Maxillofac Implants. 2003;18:894904.

21. Schwindling FS, Schwindling FP. Mini dental implants retaining mandibular overdentures: A dental practicebased retrospective analysis. J Prosthodont Res. 2016;60(3):193-8.

22. Misch CE. Short dental implants: a literature review and rationale for use. Dent Today. 2005;24:64-8.

23. Pierrisnard L, Renouard F, Renault $P$, et al. Influence of implant length and bicortical anchorage on implant stress distribution. Clin Implant Dent Relat Res. 2003;5:254-62.

24. Mahon JM, Norling BK, Phoenix RD. Effect of varying fixture width on stress and strain distribution associated with an implant stack system. Implant Dent. 2000;9:310-20.

25. Peixoto HE, Camati PR, Faot F, et al. Rehabilitation of the atrophic mandible with short implants in different positions: A finite elements study. Mater Sci Eng C Mater Biol Appl. 2017;80:122-8.

26. Pimentel MJ, Silva WJ, Del Bel Cury AA. Short implants to support mandibular complete dentures - photoelastic analysis. Braz Oral Res. 2017;31:e18.

27. Maniewicz S, Buser R, Duvernay E, et al. Short dental implants retaining two-implant mandibular overdentures in very old, dependent patients: Radiologic and clinical observation up to 5 years. Int J Oral Maxillofac Implants. 2017;32(2):415-22.

\section{ACKNOWLEDGEMENTS}

The authors would like to thank the Dentalmaia ${ }^{\circledR}$ prosthetics laboratory and MINEC (MegaGen International Network of Education and Clinical Research) for their technical and scientific support in the design and development of this technology.

\section{PEER REVIEW}

Not commissioned. Externally peer reviewed.

\section{CONFLICTS OF INTEREST}

The authors declare that they have no competing interests.

\section{FUNDING}

None

\section{PATIENT CONSENT}

The authors, Silva AS, Sá J, Aroso C, Santos E, Mendes JM, declare that:

1. They have obtained written, informed consent for the publication of the details relating to the patient(s) in this report.

2. All possible steps have been taken to safeguard the identity of the patient(s).

3. This submission is compliant with the requirements of local research ethics committees. 
Figure 1: A. Orthopantomography showing dense compact bone in the anterior mandibular region. B. Mandibular computerized axial tomography (CAT). C. Bone axial slices (CAT) between the mental foramen

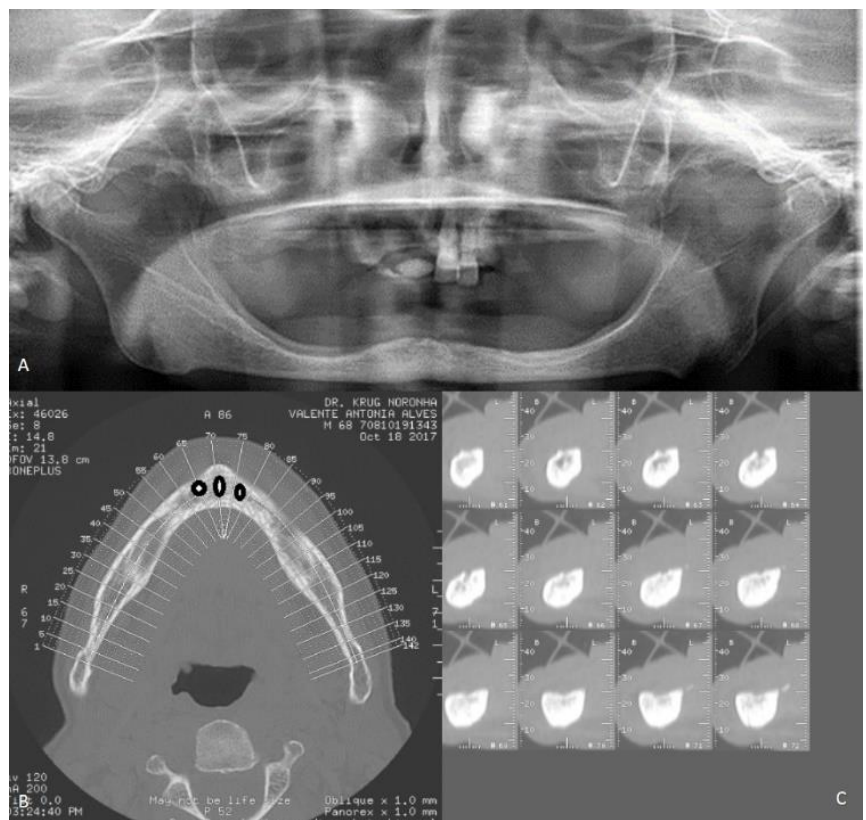

Figure 2: A. Full thickness crestal incision. B. Short implant bone insertion. $C$. Three Megagen Anyridge, $4.0 \times 8.5 \mathrm{~mm}$
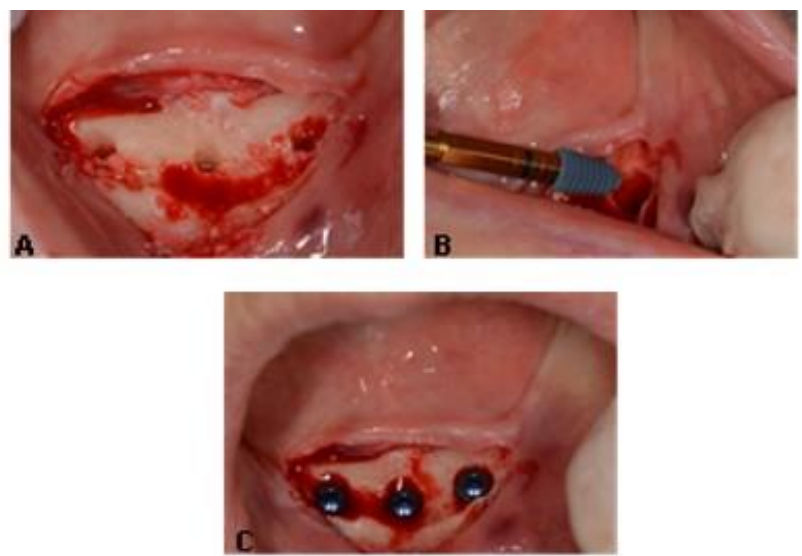

Figure 3: A. Keratinized gingiva without any sign of crestal bone loss. B. Orthopantomography performed twelve weeks post-surgery showing osseointegrated implants
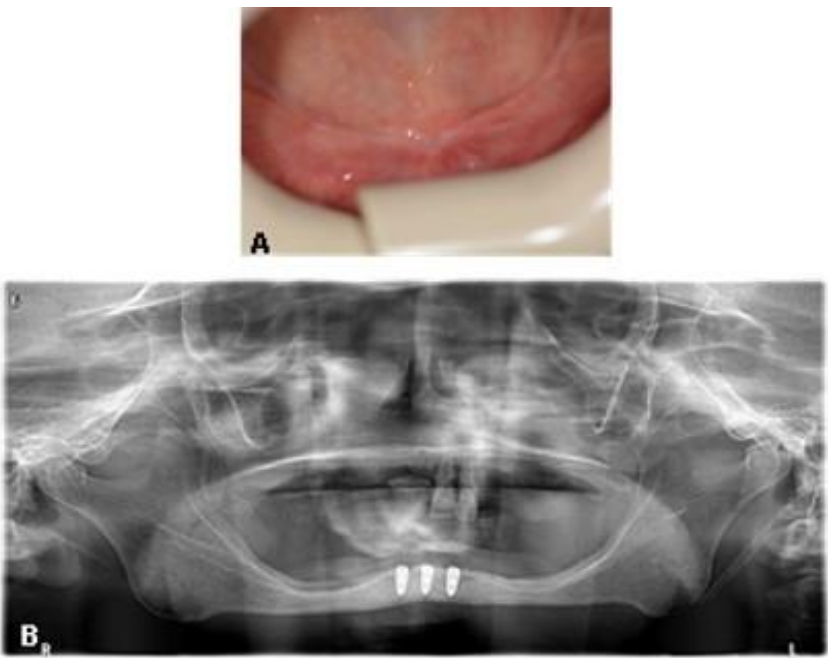

Figure 4: Healing abutment screwed into the implant body

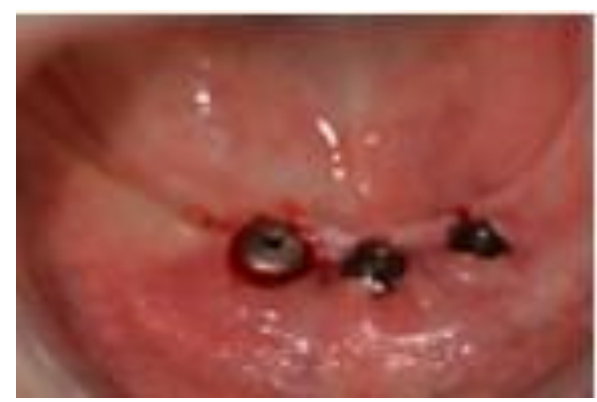

Figure 5: A. Closed tray impression copings. B. Impression made directly to the implants with a standard tray and a mixture of silicone putty and light
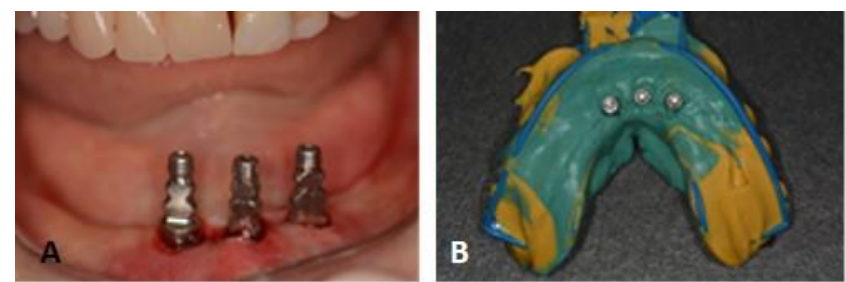
Figure 6: Occlusion wax bolted to the implant

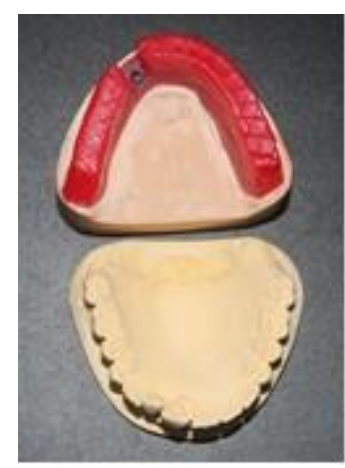

Figure 7: A. Patient's bite registration and middle and horizontal line registration with the OneBite ${ }^{\circledR}$ System B. Transfer of records to a semi-adjustable articulator
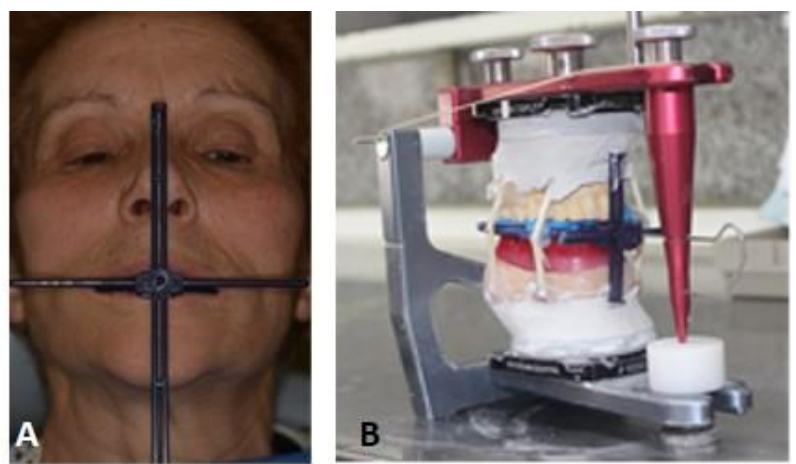

Figure 8: Screwed- in teeth test

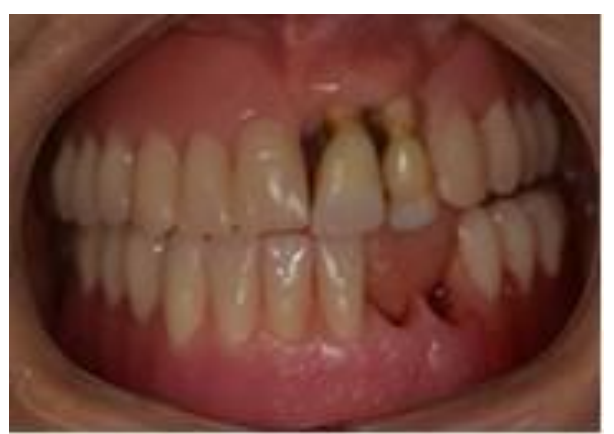

Figure 9: A. Ceroplasty for bar design. B. Test for the chrome-cobalt bar. C. Orthopantomography showing passive settlement of the bar
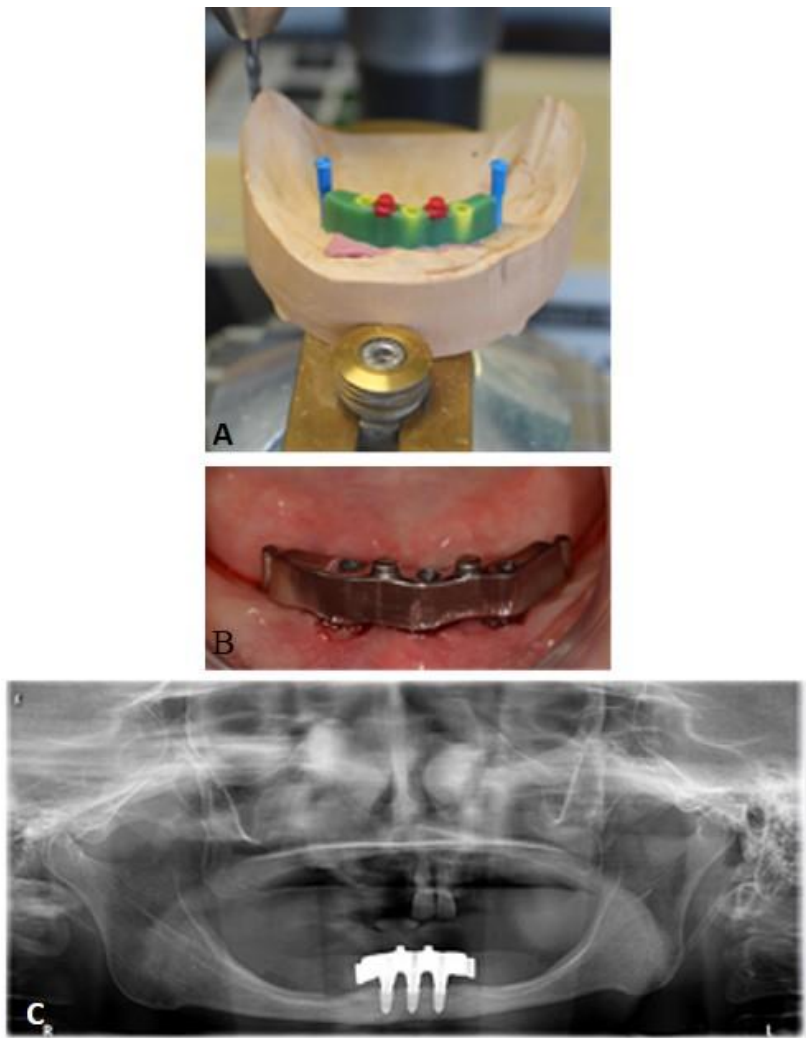

Figure 10: A. Fused reinforcement of the future prosthesis. B. Future prosthesis acrylics with metallic reinforcement. C. Prosthesis with reinforcement plus customised bar A
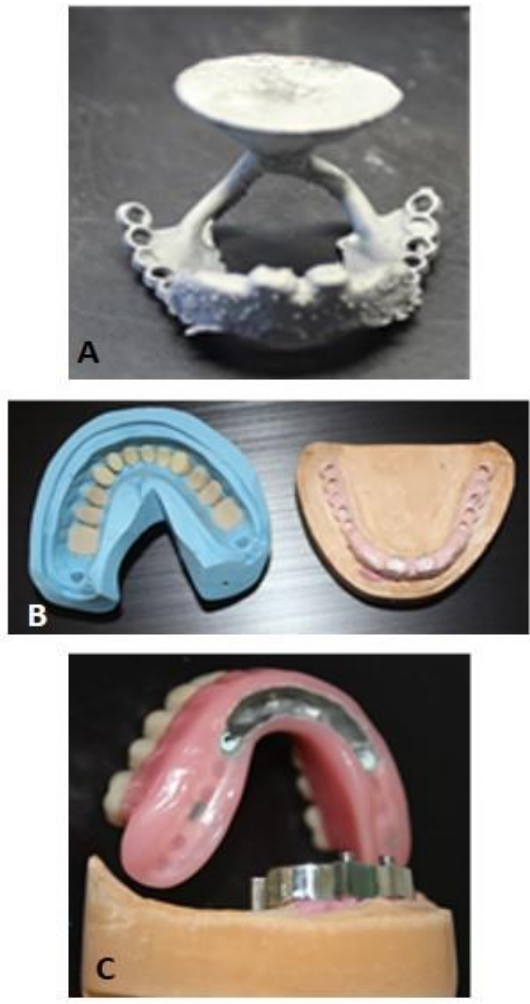
Figure 11: A. Final front view of oral rehabilitation. B. Right side view. C. Left side view. D. Final orthopantomography with dental implants, personalized bar, and prosthesis with metallic reinforcement
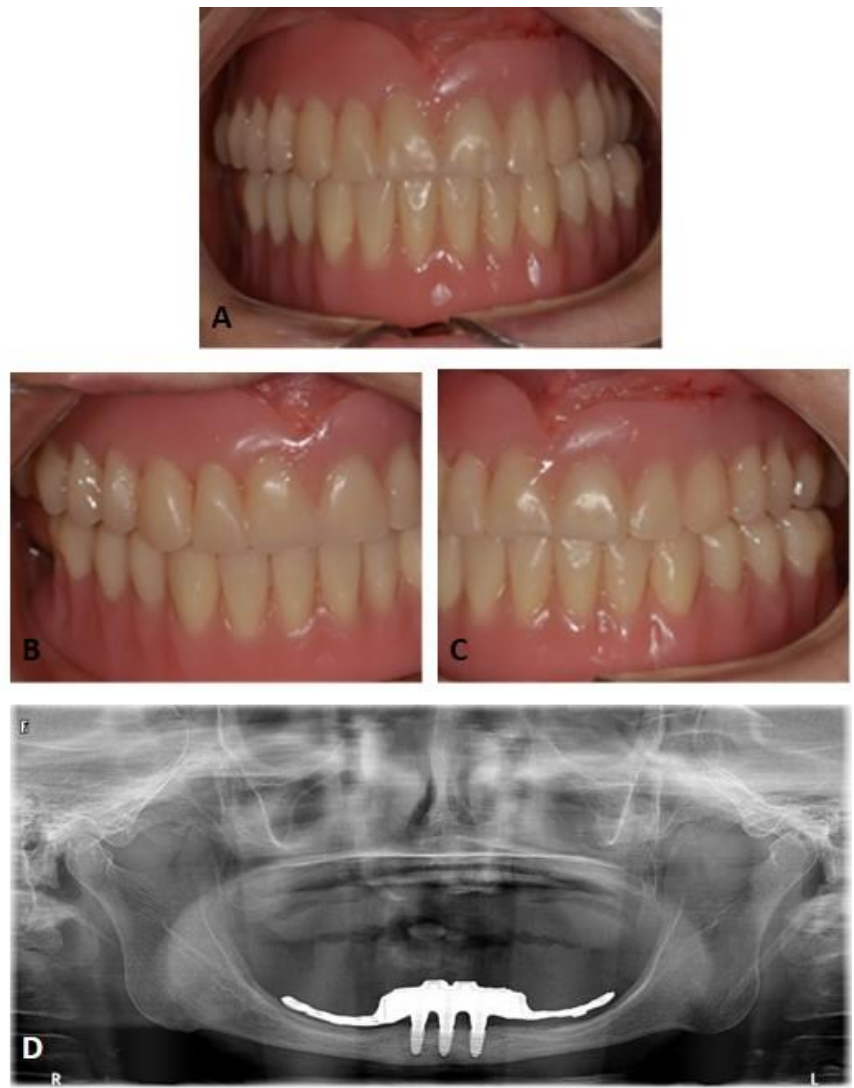